\title{
Mycological Profile of Bronchial Wash Specimens in Patients with Lower Respiratory Tract Infections
}

\author{
C.S. Sripriya*, S. Thasneem Banu, R. Deepa and N. Ratnapriya
}

Institute of Microbiology, Madras Medical College, Chennai-600003, Tamil Nadu, India

*Corresponding author

\begin{tabular}{|c|c|}
\hline & A B S T R A C T \\
\hline & \multirow{6}{*}{$\begin{array}{l}\text { Lower respiratory tract Infections (LRTIs) remains the most common infections seen in } \\
\text { the community and among hospitalized patients. The increased prevalence of fungal lung } \\
\text { infections is largely seen both in immunocompromised and immunocompetent patients. } \\
\text { Most of the fungal infections are due to Candida species, especially Candida nonalbicans } \\
\text { and among filamentaous fungi, Asperigillus spp is most common, caused by Aspergillus } \\
\text { fumigatus, A. flavus, A. niger, A. clavatus, and A. nidulans, followed by other fungal } \\
\text { organisms. The aim of the study is to isolate and speciate the different fungal pathogens } \\
\text { from bronchial wash specimens from immunocompetent and immunocompromised } \\
\text { patients with lower respiratory tract infections by conventional methods. This is a } \\
\text { prospective study conducted for a period of } 18 \text { months from January } 2015 \text { to June } 2016 \text { at } \\
\text { Institute of Microbiology, Madras Medical College. } 488 \text { bronchial wash specimens were } \\
\text { collected from patients with lower respiratory tract infections. Age of patients ranged from } \\
12 \text { years to } 80 \text { years; } 234 \text { were males and } 154 \text { were females. Fungal growth was observed } \\
\text { in } 146 \text { patient samples ( } 29.9 \% \text { ). Yeasts were isolated in } 71 \text { samples ( } 48.6 \% \text { ) and } \\
\text { filamentous fungi were isolated in } 75 \text { samples (51.3\%). Diagnosis of deep seated fungi } \\
\text { from lower respiratory tract will be more appropriate from Bronchial wash specimens than } \\
\text { sputum specimens as the rate of contamination with normal throat commensals is low and } \\
\text { isolation of true pathogens is more.Early diagnosis of deep seated fungal infections leads } \\
\text { to better control of underlying diseases and results in prolonged survival. }\end{array}$} \\
\hline Keywords & \\
\hline $\begin{array}{l}\text { Lower respiratory tract } \\
\text { Infections (LRTIs), } \\
\text { Bronchial wash, } \\
\text { Candida species, } \\
\text { Aspergillus spp. }\end{array}$ & \\
\hline Article Info & \\
\hline & \\
\hline & \\
\hline
\end{tabular}

\section{Introduction}

Lower respiratory tract Infections (LRTIs) remains the most common infections seen in the community and among hospitalized patients. The common LRTIs include Bronchitis, Bronchiolitis, and Pneumonia and Pleural infections. Though bacterial infections of the lower respiratory tract are predominant, fungal infections occur in immunocompetent and many immunocompromised states, which predisposes to them (Velez et al., 2007). Fungal lung infections are frequently encountered by pulmonary and critical care practitioners. The increased prevalence of fungal lung infections is largely related to increased numbers of immunocompromised and susceptible patients, heightened awareness of these infections, and improved laboratory methods for the diagnosis of fungal infection (Singh, 2001). Diagnosis of deep seated fungal infections in lung parenchyma can be made easier by bronchoscopy and collection of bronchial wash specimens for better isolation of fungal pathogens. Bronchial wash specimens are collected at 
bronchoscopy and submitted for evaluation of bronchial fluids, often as an adjunct to bronchial brushing and biopsy. Whereas the brush and biopsy specimens are directed at a specific target, the wash can provide a broader sample of the bronchial pathology, including organisms.

Bronchial washings are the secretions aspirated back through the bronchoscope channel after instillation of saline into a major airway. The secretions obtained by this method do not represent material from the bronchiolar or alveolar level. In intubated patients, bronchial washings are no different than an ETA obtained with a suction catheter (Abdolmajid et al., 2006)

Invasive fungal infections are a growing problem in critically ill patients and are associated with increased morbidity and mortality. Most of them are due to Candida spp, especially Candida albicans. Invasive candidiasis includes candidaemia, disseminated candidiasis with deep organ involvement and chronic disseminated candidiasis (Paramythiotou et al., 2014).

Aspergillus spores are ubiquitous in the environment and can be cultured from grain, moist soil, and vegetation, or the organism can be a laboratory contaminant. While there are more than 300 species of the organism, most human infections are caused by Aspergillus fumigatus, followed by A. flavus, A. niger, A. clavatus, and A. nidulans (Levy et al., 1992).

During the last decades, rare pathogenic fungi, such as Penicillium spp, Zygomycetes, Fusarium species and Scedosporium, Trichoderma spp have also emerged (Walsh et al., 2004).

Isolation of fungus from clinical specimen helps the physician to make empirical choice of antifungal drugs for treatment. Factors related to patient's clinical situation and present co-morbidities, local epidemiology data and purpose of treatment (prophylactic, pre-emptive, empiric or definitive) should be taken into account for the appropriate choice of antifungal agent (Paramythiotou et al., 2014).

\section{Aims and objectives}

The aim of the study is to isolate and speciate the different fungal pathogens from bronchial wash specimens from immunocompetent and immunocompromised patients with lower respiratory tract infections.

\section{Materials and Methods}

This is a prospective study conducted for a period of 18 months from January 2015 to June 2016 at Institute of Microbiology, Madras Medical College. 488 bronchial wash specimens were collected from patients with lower respiratory tract infections.

Fungal cultures and identification were performed on fresh specimens by standard procedures in microbiology laboratory. The specimens were subjected to $\mathrm{KOH}$ mount for initial identification and cultured on Sabouraud Dextrose Agar with antibiotics. The inoculated specimens were incubated at $37^{\circ} \mathrm{C}$ and $25^{\circ} \mathrm{C}$.

Yeast-form fungi were identified according to standard clinical laboratory methods, including the grams staining, germ tube test and Chromagar identification. Mould-form fungi were identified using colony morphology and microscopic findings.

\section{Results and Discussion}

A total of 488 bronchial wash specimens were collected from patients with lower respiratory 
tract infections. Age of patients ranged from 12 years to 80 years; 234 were males and 154 were females (Fig. 1).

Fungal growth was observed in 146 patient samples $(29.9 \%)$. Yeasts were isolated in 71 samples $(48.6 \%)$ and filamentous fungi were isolated in 75 samples (51.3\%) (Fig. 2).

Candida non-albicans predominated yeasts, with 23 isolates of Candida tropicalis, 14 isolates of Candida glabrata and 8 isolates of Candida krusei. Candida albicans was isolated in 9 samples (Fig. 3).

Filamentous fungi were isolated in 75 $(51.3 \%)$ samples. Identification was done based on macroscopic colony morphology and microscopic identification. Aspergillus species was the predominant fungi isolated in bronchial wash specimens, Penicillium spp was isolated in 4 specimens and Paecillomyces spp in 4 specimens. Rhizopus spp, Rhizomucor spp, Pseudallerichia boydii, were isolated from one specimen each (Fig. 4).

Aspergillus fumigates was the predominant isolate among Aspergillus spp, with 25 Aspergillus fumigates isolated from bronchial wash samples. Aspergillus flavus was isolated from 12 samples, Aspergillus nidulans from 3 samples, Aspergillus niger from 16 samples, Aspergillus terreus from 6 samples and Aspergillus versicolor from 2 samples (Fig. 5).

Respiratory tract infections are common cause of hospitalisation. In this study we evaluated the overall prevalence of fungal infections in lower respiratory tract (Yazicioglu Mocin et al., 2013)

Fig.1 Male vs. female ratio

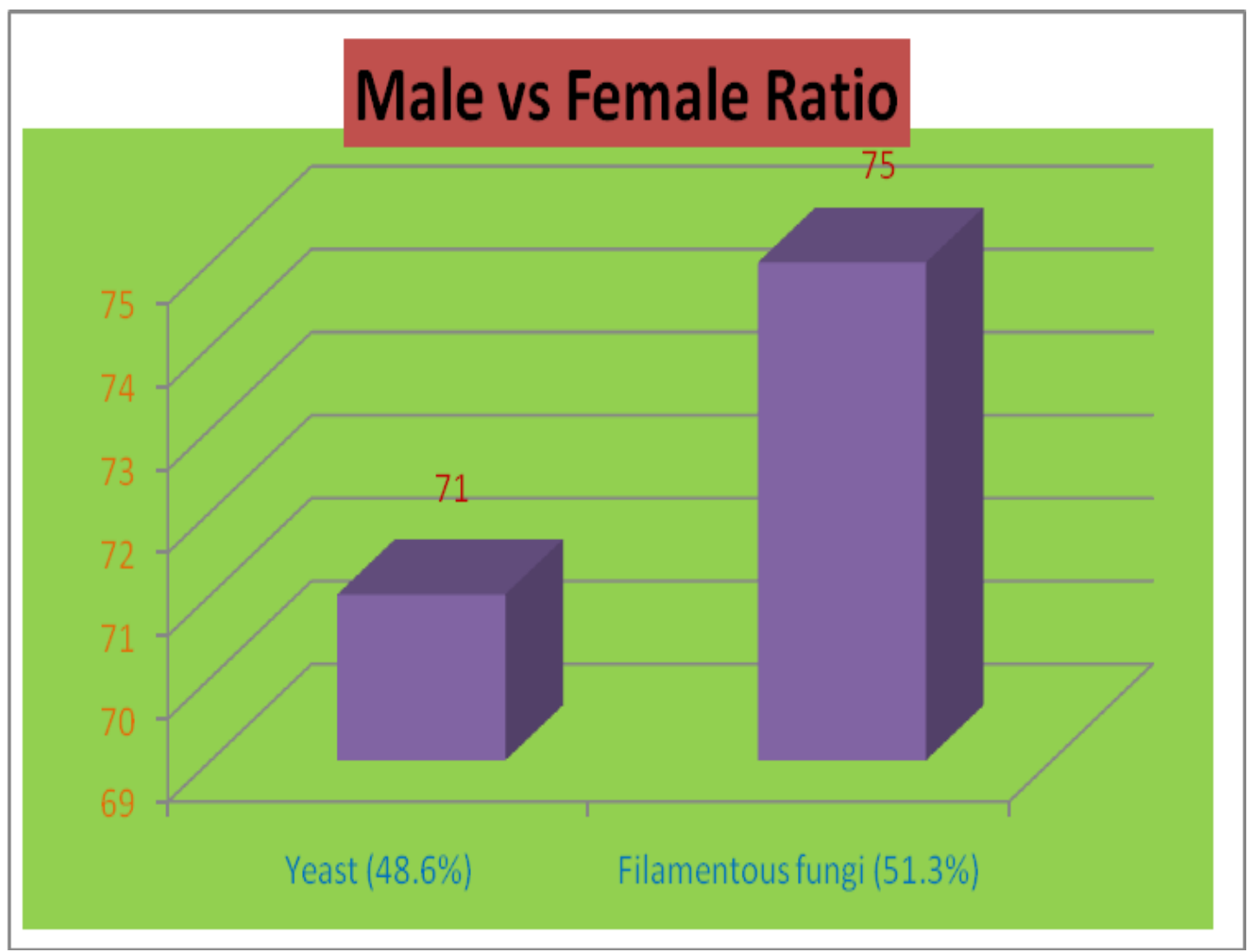


Fig.2 Fungal growth pattern in bronchial wash specimens

\section{Growth pattern of Fungal Isolates}

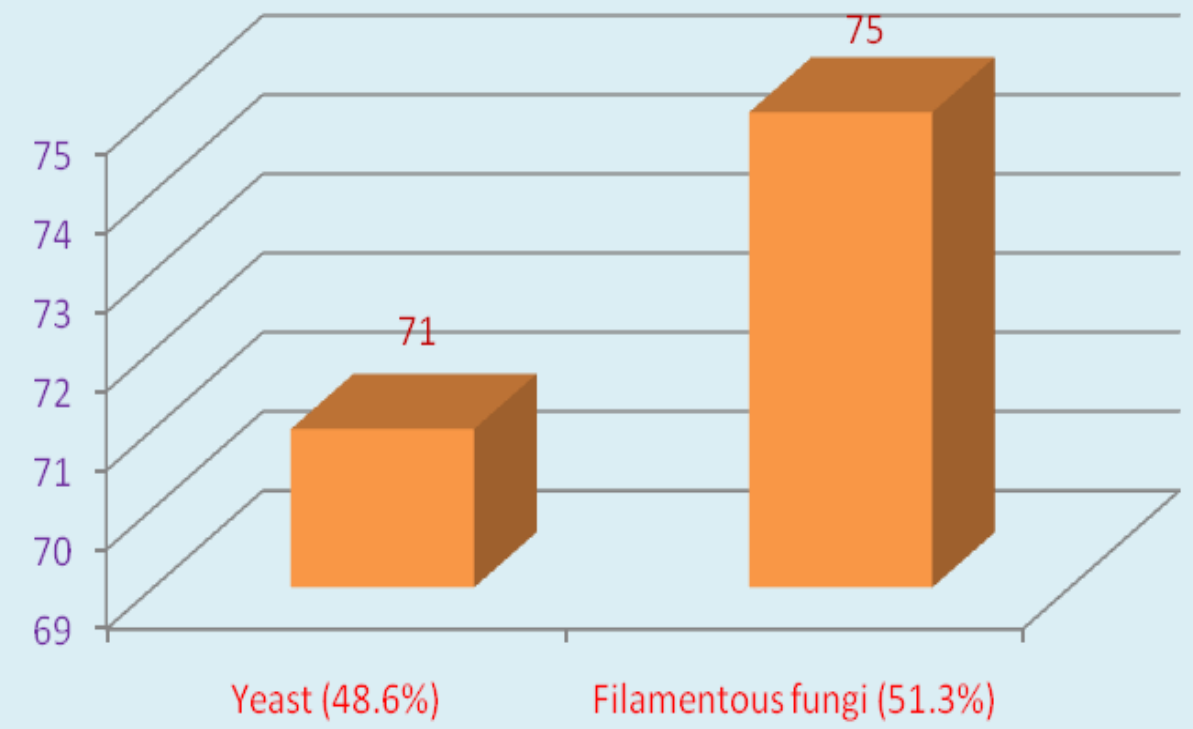

Fig.3 Yeats identified in bronchial wash samples

\section{No of Yeast Isolates}

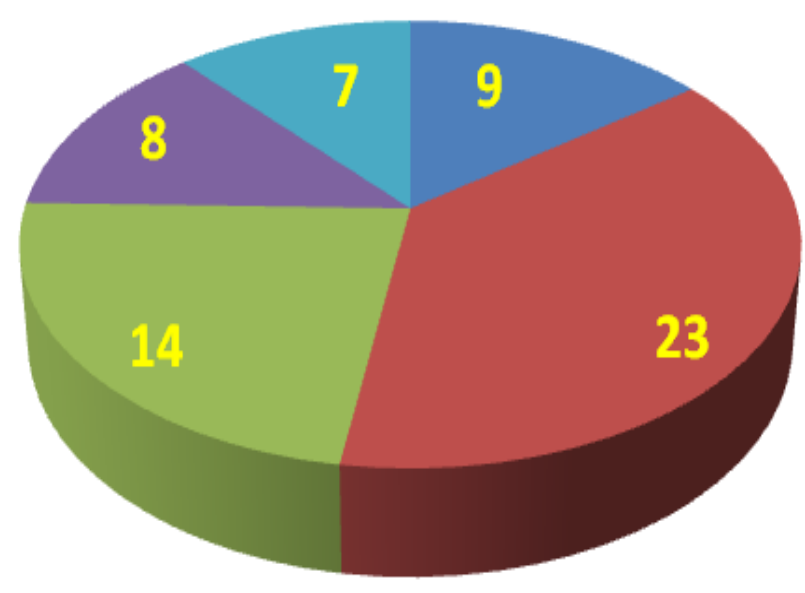

C. albicans

C. tropicalis

C. glabrata

C. krusei

Other non albicans candida 
Fig.4 Distribution of other fungal isolates

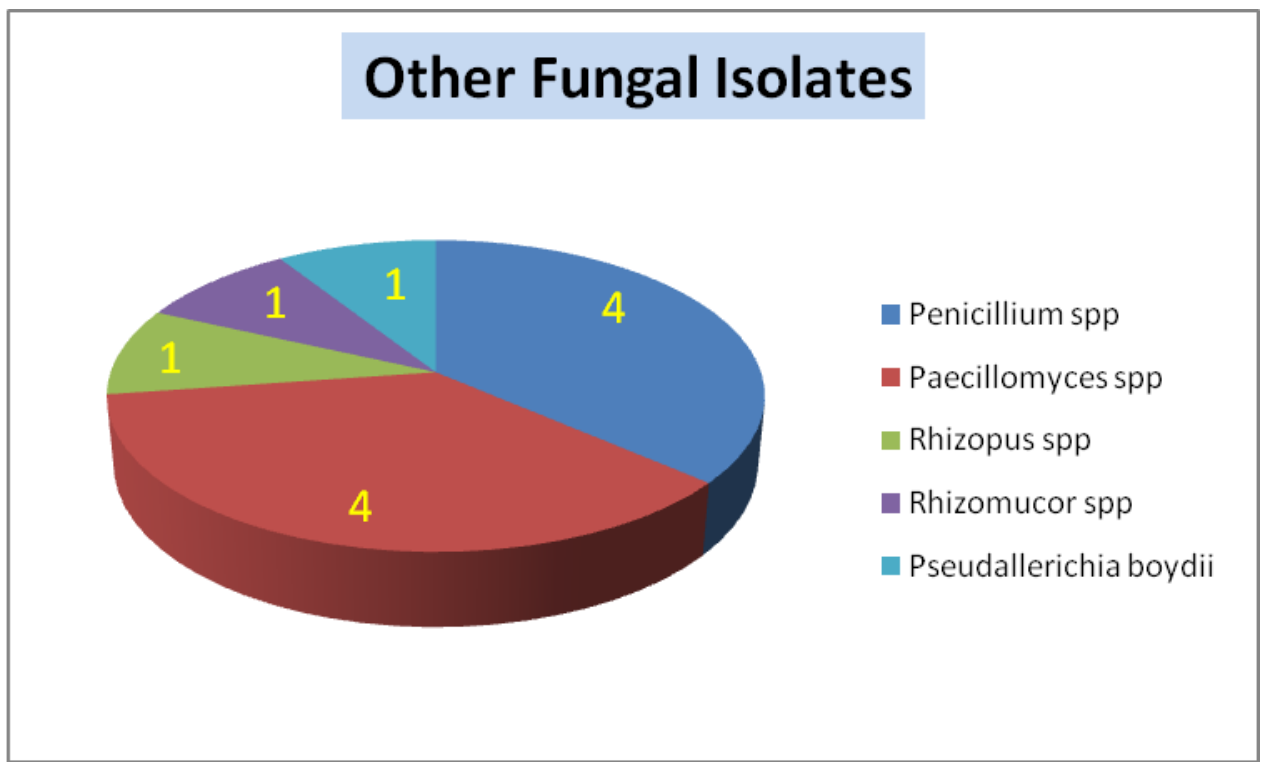

Fig.5 Distribution of Aspergillus species in Bronchial wash specimens

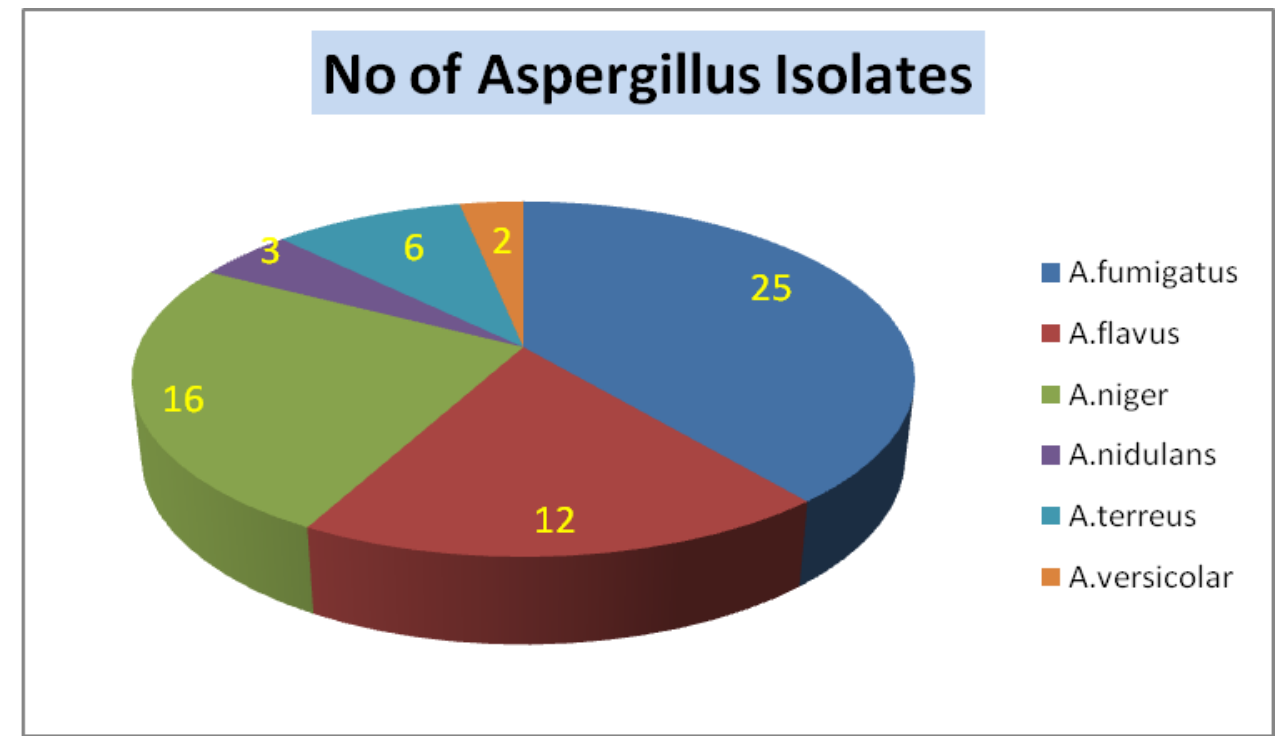

Though bacterial etiology of lower respiratory tract is more common, fungal infections are increasingly reported in both immunocompetent and immunocompromised patients.

In this study, Males were found to be more affected $(47.9 \%)$ than females $(31.5 \%)$. An increased incidence of yeasts was found than filamentous fungi. The increased proportion of non-albicans species has been also observed in critically ill patients with considerable differences in the percentage of albicans vs. non-albicans spp (Falagas et al., 2010). In an Italian study $40 \%$ of the fungaemia episodes were due to $C$. albicans, followed by $C$. parapsilosis (23\%), $C$. glabrata (15\%), C. tropicalis (9\%) and other species (13\%) (Bassetti et al., 2006). Nonalbicans Candida was found to be more in 
lower respiratory tract infections than Candida albicans in our study.

Among the hundreds of Aspergillus species few are able to cause disease to humans. The most commonly encountered include $A$. fumigatus followed by $A$. flavus and $A$. terreus. The epidemiology of Aspergillosis in the ICU is difficult to establish due to the inhomogeneity of hospitalised patients, the diagnostic difficulties necessitating a biopsy and the difficulty in discriminating between colonisation and disease (Meersseman et al., 2007), which was also seen from our study.

Invasive fungal infections are increasingly common among the lower respiratory tract infections in the community and nosocomial setting.

Isolating deep seated fungal infections from distal part of the lung will be of immense use in diagnosis and treatment of the same.

Diagnosis of deep seated fungi from lower respiratory tract will be more appropriate from Bronchial wash specimens than sputum specimens as the rate of contamination with normal throat commensals is low and isolation of true pathogens is more.

Furthermore, because risk-factors associated with these infections continue to increase in frequency, it is likely that the incidence of fungal infections will continue to increase in the coming decades. This expansion is based on an increase in the number of immunocompromised patients, including cancer patients with chemotherapy-induced neutropenia, transplant recipients receiving immunosuppressive therapy, and human immunodeficiency virus infected patients.

Early diagnosis of deep seated fungal infections leads to better control of underlying diseases and results in prolonged survival.

\section{References}

Abdolmajid, F., Majid, M., Fariba, R., Fariba, B., Sara, F. 2006. Fungal Infection in Samples of Bronchial washing in the Department of Bronchoscopy in Emam Reza Hospital of Mashhad in 20042005. Medical Science Journal, 13, 8389.

Bassetti, M., Righi, E., Costa, A., Fasce, R., Molinari, M. P., Rosso, R., Viscoli, C. 2006. Epidemiological trends in nosocomial candidemia in intensive care. BMC infectious diseases, 6(1), 21.

Falagas, M. E., Roussos, N.,Vardakas, K. Z. 2010. Relative frequency of albicans and the various non-albicans Candida spp among candidemia isolates from inpatients in various parts of the world: a systematic review. International Journal of Infectious Diseases, 14(11), e954-e966.

Levy, H., Horak, D. A., Tegtmeier, B. R., Yokota, S. B., Forman, S. J. 1992. The value of bronchoalveolar lavage and bronchial washings in the diagnosis of invasive pulmonary aspergillosis. Respiratory medicine, 86(3), 243-248.

Meersseman, W., Lagrou, K., Maertens, J., Wijngaerden, E. V. 2007. Invasive aspergillosis in the intensive care unit. Clinical Infectious Diseases, 45(2), 205216.

Paramythiotou, E., Frantzeskaki, F., Flevari, A., Armaganidis, A., Dimopoulos, G. 2014. Invasive fungal infections in the ICU: how to approach, how to treat. Molecules, 19(1), 1085-1119.

Singh, N. Trends in the epidemiology of opportunistic fungal infections: predisposing factors and the impact of antimicrobial use practices. Clin Infect Dis, 2001; 33:1692-1696.

Velez, L., Correa, L. T., Maya, M. A., Mejia, P., Ortega, J., Bedoya, V., Ortega, H. 2007. Diagnostic accuracy of 
bronchoalveolar lavage samples in immunosuppressed patients with suspected pneumonia: analysis of a protocol. Respiratory medicine, 101 (10), 2160-2167.

Walsh, T. J., Groll, A., Hiemenz, J., Fleming, R., Roilides, E., Anaissie, E. 2004. Infections due to emerging and uncommon medically important fungal pathogens. Clinical Microbiology and
Infection, 10(s1), 48-66. Yazicioglu Mocin, O., Karakurt, Z., Aksoy, F., Güngör, G., Partal, M., Adıgüzel, N., Erdem, H. 2013. Bronchoscopy as an indicator of tracheobronchial fungal infection in non- neutropenic intensive- care unit patients. Clinical Microbiology and Infection, 19(3).

\section{How to cite this article:}

Sripriya, C.S., S. Thasneem Banu, R. Deepa and Ratnapriya, N. 2017. Mycological Profile of Bronchial Wash Specimens in Patients with Lower Respiratory Tract Infections. Int.J.Curr.Microbiol.App.Sci. 6(11): 176-182. doi: https://doi.org/10.20546/ijcmas.2017.611.022 